\title{
Microembolic signal counts increase during hyperbaric exposure in patients with prosthetic heart valves
}

\author{
R. W. Baumgartner, MD \\ A. Frick, MD \\ C. Kremer, $\mathrm{MD}^{\mathrm{a}}$ \\ E. Oechslin, $M D^{\mathrm{b}}$ \\ E. Russi, $M^{\mathrm{c}}$ \\ J. Turina, $M D^{b}$ \\ D. Georgiadis, MD
}

From the Department of Neurology and the Divisions of Cardiology and Pneumology, ${ }^{\mathrm{c}}$ Department of Internal Medicine, University of Zurich, Switzerland, and the Department of Neurology, ${ }^{\mathrm{d}}$ University of Heidelberg, Germany.

Received for publication March 1, 2001; revisions requested March 28, 2001; revisions received May 7, 2001; accepted for publication May 17, 2001.

Address for reprints: Ralf W. Baumgartner, MD, Department of Neurology, University Hospital, Frauenklinikstrasse 26, CH-8091 Zurich, Switzerland (E-mail: ralf.baumgart ner@nos.usz.ch).

J Thorac Cardiovasc Surg 2001;122:1142-6

Copyright (C) 2001 by The American Association for Thoracic Surgery

$0022-5223 / 2001 \$ 35.00+0 \quad \mathbf{1 2 / 1 / 1 1 7 2 8 2}$

doi:10.1067/mtc.2001.117282
Background: Patients with prosthetic heart valves have an increased risk of thromboembolic events, and transcranial Doppler sonography reveals microembolic signals. Whereas microembolic signals were initially assumed to be of particulate matter, recent studies suggest that they are partially gaseous in origin. If this is true, alteration of environmental pressure should change microembolic signal counts. We undertook this study to evaluate the influence of hyperbaric exposure on microembolic signal counts in persons with prosthetic heart valves.

Methods and Results: Microembolic signal counts were monitored by transcranial Doppler sonography of both middle cerebral arteries under normobaria (normobaria 1), 2 subsequent periods of hyperbaria ( 2.5 and 1.75 bar), and a second period of normobaria (normobaria 2) in 15 patients with prosthetic heart valves. Each monitoring period lasted 30 minutes. Compression and decompression rates were $0.1 \mathrm{bar} / \mathrm{min}$. Microembolic signal counts increased from 20 (12-78) at normobaria 1 to 79 (30-165) at $2.5 \mathrm{bar}(P<.01$ vs normobaria 1 and 2$)$, decreased to $44(18-128)$ at $1.75 \mathrm{bar}(P<$ .01 vs normobaria 1 and 2.5 bar; $P<.001$ vs normobaria 2$)$, and returned to 20 (8-96) at normobaria 2 (values are medians and $95 \%$ confidence intervals).

Conclusions: Our results strongly suggest that gaseous bubbles are underlying material for part of the microembolic signals detected in patients with prosthetic heart valves.

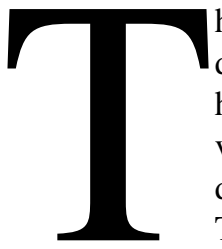

he implantation of prosthetic heart valves represents an important development in cardiac surgery because the valves improve the hemodynamic function of the heart. Unfortunately, prosthetic heart valves are associated with thromboembolic events, which occur despite adequate anticoagulation in $0.8 \%$ to $5.1 \%$ per patient-year. ${ }^{1-5}$ The risk is cumulative over time, involves the cerebral circulation in more than $85 \%$ of cases, and leads to a permanent neurologic deficit in $50 \%$ and to death in $10 \%$. $2,6,7$

Monitoring for microembolic signals (MES) by means of transcranial Doppler sonography is a validated technique for detection of embolic material in the insonated sample volume. ${ }^{8,9}$ To date, MES detection has been described in several stroke-prone patient groups, including patients with artificial heart valves. ${ }^{10-13}$ The clinical relevance of MES in the latter group remains a matter of debate. At the same time, unequivocal evidence concerning the underlying embolic material is still lacking. Recently reported significant changes in MES counts during inhalation of $100 \%$ oxygen suggest that at least part of MES are caused by nitrogen bubbles. ${ }^{14}$ If this were true, hyperbaria would be expected to influence the number of MES. Up 
to now, 3 patients with prosthetic heart valves have been examined under hyperbaric conditions with conflicting results, because the number of MES increased in 2 patients but remained unchanged in the other. ${ }^{15,16}$

The purpose of the present hyperbaric chamber study was to investigate MES counts in patients with prosthetic heart valves during exposure to 2 different levels of hyperbaria (2.5 and 1.75 bar) and to compare them with MES counts measured at normobaria.

\section{Patients and Methods Patients}

Thirty-five patients with prosthetic heart valves and no history of thromboembolic disease were recruited from the Department of Cardiothoracic Surgery and the Division of Cardiology, Department of Internal Medicine, University of Zurich, Switzerland. During the study the anticoagulation levels were within the accepted norms for the prostheses used. Twenty patients were included in the study, because 15 patients were excluded on the basis of the criteria mentioned below. All exclusions resulted from scuba diving criteria, whereas no patient was excluded because of ultrasonic criteria.

Inclusion criteria were the presence of artificial heart valves, at least one temporal acoustic window providing adequate spectral Doppler signals from the middle cerebral artery (MCA), and no ultrasonographic evidence of stenosis or occlusion of extracranial and intracranial cerebral arteries.

Internationally accepted exclusion criteria for scuba diving were applied to avoid adverse effects resulting from hyperbaria. These criteria were history of overpressure accident in previous diving, history of nervous system disease including seizure disorder, psychiatric disease including substance and drug abuse, coryza, bronchitis, inability to equalize pressure in the middle ear by autoinflation, perforation of the tympanic membrane, middle ear surgery with placement of a prosthesis in the conduction chain, symptomatic coronary artery disease, bullous lung disease, significant obstructive pulmonary disease, history of spontaneous pneumothorax, abdominal hernia, and pregnancy. ${ }^{17}$

Stenosis or occlusion of cerebral arteries may cause MES. Therefore, all patients were evaluated by extracranial and transcranial color duplex sonography, and the presence of cerebral artery stenosis or occlusion was another exclusion criterion.

Written informed consent was obtained from all patients, and our study protocol was reviewed and approved by the Ethical Committee of the University Hospital in Zurich.

\section{Ultrasonic Examinations}

The common, internal, and external carotid arteries and the subclavian and vertebral arteries were investigated with the use of a color duplex scanner (Acuson Sequoia, Mountain View, Calif) equipped with a linear 4- to 8-MHz probe. Transorbital insonation of the ophthalmic arteries and the carotid siphon was performed by means of a transcranial Doppler device (TC2-64B, EME, Überlingen, Germany) equipped with a 2-MHz probe. Transtemporal and transoccipital insonation of the anterior, middle, and posterior cerebral arteries and the intracranial vertebral and basilar arteries was done with a $2.0-$ to $3.5-\mathrm{MHz}$ sector probe attached to the same color duplex scanner used for extracranial insonation. Examination and assessment of cerebral artery stenosis and occlusion were performed as reported previously. ${ }^{18-21}$

\section{Hyperbaric Chamber Study}

The hyperbaric chamber of the Division of Pneumology, Department of Internal Medicine, University of Zurich, was used for this study. Because of place restriction, each exposure to hyperbaria was performed with a maximum of 2 patients under the surveillance of 1 examiner in the chamber as well as 2 members of the staff of the hyperbaric chamber who were performing compression and decompression. The patients were examined in the supine position. The MCA was insonated bilaterally through the temporal acoustic window, and both 2-MHz transducers were fixed on the skull with an elastic band to minimize movement artifacts. Two pulsed-wave Doppler machines (Multi-Dop X; DWL, Sipplingen, Germany) were located outside the hyperbaric chamber. The ultrasound devices were connected to the transducers by a cable, which penetrated the wall of the chamber through a pressure-stable valve. Persons within and outside the chamber were in contact by intercom.

The number of MES and the heart rate (HR) were counted in both MCAs under normobaria (normobaria 1) for 30 minutes. Subsequently, both patients and the investigator were compressed to 2.5 bar, which corresponds to a sea water depth of $15 \mathrm{~m}$. The rate of compression was $0.1 \mathrm{bar} / \mathrm{min}$. MES and HR were registered in both MCAs for 30 minutes under stable hyperbaria of 2.5 bar. Thereafter, the patients and the investigator were decompressed at a rate of $0.1 \mathrm{bar} / \mathrm{min}$ to $1.75 \mathrm{bar}$, which corresponds to a sea water depth of $7.5 \mathrm{~m}$. Under stable hyperbaria of 1.75 bar, MES and HR were registered in both MCAs for 30 minutes. Final decompression to normobaria (normobaria 2) was done at a rate of 0.1 bar/min, and MES and HR were registered in both MCAs for 30 minutes under normobaria.

\section{Microembolic Signal Detection}

The MCA was insonated at 2 spatially separated depths (distal sample volume at 46 to $52 \mathrm{~mm}$, proximal sample volume at 52 to $57 \mathrm{~mm}$ ). The distance between the 2 sample volumes was set at 5 $\mathrm{mm}$. A 64-point fast Fourier transformation (FFT) was used with an FFT length of $2 \mathrm{~ms}$ and an FFT overlap of $60 \%$. Settings of the Doppler machine were as follows: power $110 \mathrm{~mW}$, sample volume 5 to $8 \mathrm{~mm}$, low-pass filter $80 \mathrm{kHz}$, high-pass filter $100 \mathrm{~Hz}$, and sweep 5 seconds. These settings were used throughout the study.

MES were detected on the basis of the following criteria: (1) characteristic sound, (2) unidirectionality within the flow signature of the vessel monitored, (3) intensity increase at least $6 \mathrm{~dB}$ above background, and (4) random appearance in the cardiac cycle according to a recent consensus statement. ${ }^{22}$ Two human observers were present during all monitoring sessions. Additionally, all ultrasound data were harvested in an 8-channel DAT recorder (TASCAM DA 88; Tascam Corp, Los Angeles, Calif) for later re-evaluation. This was performed by assigning all tapes to another experienced observer, blinded to the identity of the patient and the period of examination. Both examiners were asked to note the exact position of each MES, based on the time display of the DAT recorder. Only signals identified as MES by both observers were 


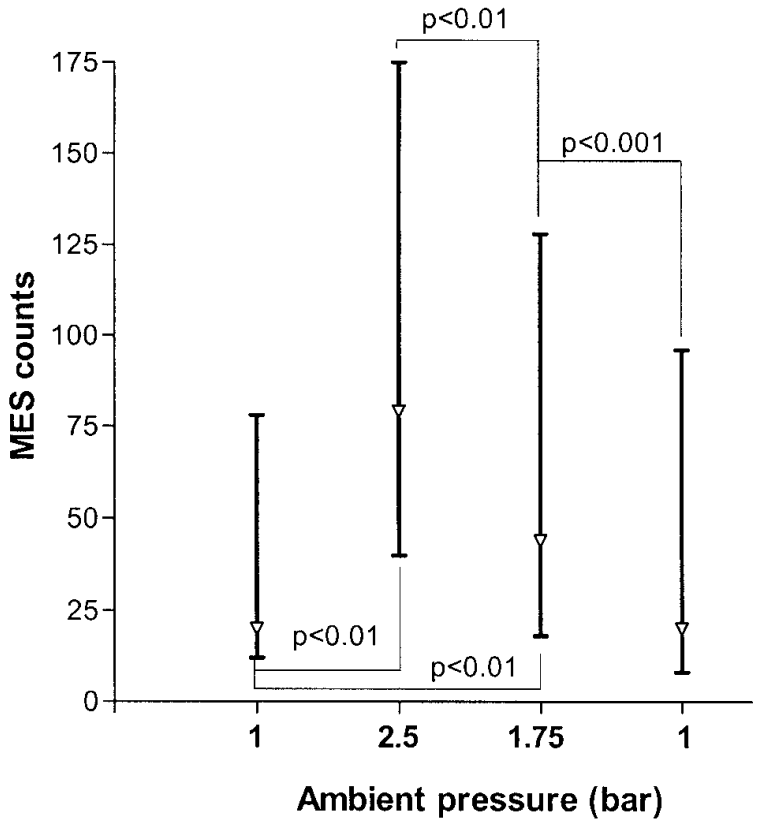

Figure 1. MES counts during normobaria, 2 subsequent levels of hyperbaria (2.5 and $1.75 \mathrm{bar}$ ), and a second period of normobaria in 15 patients with prosthetic heart valves. MES counts are expressed as medians and $95 \%$ confidence intervals and are compared by means of the Wilcoxon signed rank test (Friedman test for all 4 measurement periods, $P<.0001$ ).

further evaluated. MES counts represent the sum of both MCAs. Differences between the 2 sides were not further evaluated.

\section{Statistical Analysis}

Normally distributed data were expressed as mean \pm standard deviation; nonnormally distributed data were expressed as median and 95\% confidence intervals. MES and HR measurements obtained at both normobaric and hyperbaric periods were compared with each other by nonparametric analysis of variance by means of the Friedman test and the Wilcoxon signed rank test.

Interobserver agreement was evaluated by means of the Cohen $\kappa$ statistic. Values derived from this evaluation ranged between -1 (complete disagreement) and 1 (complete agreement), with a value of 0 reflecting a lacking relation between the evaluations of the 2 observers. Acceptable to good agreement is indicated by values between 0.4 and 0.75 ; values greater than 0.75 indicate an excellent agreement. ${ }^{23}$

\section{Results}

\section{Adverse Events and Dropouts}

Three patients had ear pain during the first meters of hyperbaria. Compression was stopped immediately, and the patients were decompressed slowly. Ear pain disappeared completely within 15 to 320 minutes. Two patients withdrew from the study. Thus, 15 patients completed the study protocol. These were 2 women and 13 men, aged $52 \pm 12$ years (range 25-75 years). They had Carbomedics (Sulzer Carbomedics, Inc, Austin, Tex; $n=13$ ) and St Jude Medical (St Jude Medical, Inc, St Paul, Minn; $n=4)$ valves in the aortic $(n=9)$, mitral ( $n$ $=4)$, or both mitral and aortic positions $(n=2)$.

\section{Microembolic Signal Counts (Figure 1 and Table 1)}

During normobaria 1 the number of detected MES was 20 (12-78). During hyperbaria of 2.5 bar the number of MES increased to $79(30-165 ; P<.01$ vs normobaria 1$)$. The number decreased to 44 (18-128) during hyperbaria of 1.75 bar $(P<.01$ vs normobaria 1 and hyperbaria of 2.5 bar). MES counts decreased during normobaria 2 to 20 (8-96) and were lower than the counts recorded during hyperbaria of 2.5 bar $(P<.01)$ and 1.75 bar $(P<.001)$ (Friedman test: $P<.001$; values are medians [95\% confidence intervals]).

\section{Heart Rate}

HR was 72 beats/min (67-80 beats/min) at normobaria 1, remained stable at 2.5 bar (70 [64-78]), decreased at 1.75 bar (68 [61-73]; $P<.01$ vs normobaria 1$)$, and increased to 71 beats/min $(66-77$ beats/min) at normobaria $2(P<.05$ vs hyperbaria of 1.75 bar) (Friedman test: $P<.01$; values are medians $[95 \%$ confidence intervals]).

\section{Interobserver Agreement}

A total of 4562 MES were detected by at least 1 observer (first observer 4420, second observer 4437). Both observers unanimously identified 4295 signals as MES. A total of 105 signals $(2.3 \%)$ were identified by 1 observer as MES and overlooked by the other one, and 152 signals (3.3\%) were identified as MES by 1 observer and as artifacts by the other. The acquired $\kappa$ value indicated excellent observer agreement $(\kappa=0.97)$.

\section{Discussion}

Although MES in patients with prosthetic valves were already described in $1990,{ }^{24}$ their underlying embolic material remained a matter of continuous debate. The lack of influence of the intensity of oral anticoagulation on MES counts, ${ }^{25}$ the lack of correlation between MES counts and D-dimers, thrombin-antithrombin complex, and antithrombin III levels, ${ }^{26}$ and the insignificant changes in MES counts despite the administration of aspirin or heparin ${ }^{27}$ essentially argued against full blood clots or activated platelets as underlying embolic material. The exponential decrease in MES counts during inhalation of $100 \%$ oxygen, which corresponded with the exponential decline in blood nitrogen concentration, suggested nitrogen gas bubbles as underlying embolic material. ${ }^{14}$ The present study adds further weight to this hypothesis: exposure to hyperbaria resulted in a significant increase in MES counts, which essentially returned to baseline values under normobaric conditions. This pattern corresponds to concordant changes in the partial pressure of 
TABLE 1. MES counts under normobaric and hyperbaric conditions in 15 patients with prosthetic heart valves

\begin{tabular}{|c|c|c|c|c|c|c|}
\hline \multicolumn{3}{|c|}{ Clinical details } & \multicolumn{4}{|c|}{ MES counts } \\
\hline Patient No. (age [y], sex) & Valve type & Valve position & 1 bar & 2.5 bar & 1.75 bar & 1 bar \\
\hline $1(64, M)$ & CM & AVR & 14 & 23 & 17 & 10 \\
\hline $2(75, M)$ & $\mathrm{CM}$ & $\mathrm{A}+\mathrm{MVR}$ & 31 & 107 & 63 & 32 \\
\hline $3(53, M)$ & SJM & MVR & 148 & 302 & 240 & 187 \\
\hline $4(25, F)$ & $\mathrm{CM}$ & MVR & 20 & 27 & 17 & 10 \\
\hline $5(39, M)$ & $\mathrm{CM}$ & AVR & 21 & 150 & 110 & 47 \\
\hline $6(38, F)$ & $\mathrm{CM}$ & MVR & 315 & 654 & 435 & 260 \\
\hline $7(50, M)$ & CM & AVR & 13 & 49 & 11 & 0 \\
\hline $8(53, M)$ & $\mathrm{CM}$ & AVR & 3 & 4 & 8 & 5 \\
\hline $9(53, M)$ & SJM & AVR & 24 & 133 & 57 & 18 \\
\hline $10(50, M)$ & $\mathrm{CM}$ & AVR & 5 & 44 & 22 & 4 \\
\hline $11(62, \mathrm{M})$ & $\mathrm{CM}$ & $\mathrm{A}+\mathrm{MVR}$ & 46 & 169 & 132 & 46 \\
\hline $12(41, M)$ & SJM & AVR & 20 & 63 & 37 & 16 \\
\hline $13(58, M)$ & $\mathrm{CM}$ & AVR & 18 & 32 & 28 & 12 \\
\hline $14(52, \mathrm{M})$ & $\mathrm{CM}$ & AVR & 1 & 4 & 7 & 3 \\
\hline $15(61, M)$ & SJM & MVR & 7 & 4 & 7 & 3 \\
\hline
\end{tabular}

CM, Carbomedics; SJM, St Jude Medical; AVR, aortic valve replacement; MVR, mitral valve replacement; A+MVR, dual (aortic and mitral) valve replacement.

nitrogen, which have been extensively described in diving literature. ${ }^{17,28}$

An increasing HR during exposure to hyperbaria might be another explanation of the raised MES counts observed in this study. At hyperbaria, however, HR either remained unchanged or decreased compared with normobaric values. These findings indicate that HR did not increase MES counts at hyperbaria.

Generation of cavitation bubbles through mechanical valves is a common occurrence in mock circulation flow loops. ${ }^{29-31}$ Still, these bubbles are supposed to implode within milliseconds ${ }^{32,33}$ and should thus be unable to enter the cerebral circulation. We hypothesize that some cavitation bubbles could be energetically more stable and thus have a longer life span than previously assumed. In particular, cavitation bubbles of bigger diameter possess a higher stability and a longer life span, which could potentially allow them to enter the systemic circulation. ${ }^{31}$ Observations on cavitation bubbles were almost solely performed under in vitro conditions, with water or normal saline solution as the circulating fluid, so that the influence of potential interactions between blood contents (particularly serum proteins) and cavitation bubbles could not be examined. Bubbles could indeed be detected several centimeters downstream from the prosthetic valve with an ultrasound probe in a recently published study, where porcine blood was used as circulating fluid in the mock circulatory loop, ${ }^{34}$ indicating a life span in the order of seconds. Therefore, the assumption that the underlying material of MES in patients with prosthetic heart valves could consist of cavitation bubbles appears more probable than initially assumed.

The number of cavitation bubbles decreases with rising static pressure. ${ }^{35}$ Hence, hyperbaria is expected to suppress cavitation and decrease the number of MES. Consequently, another mechanism such as an augmented concentration of blood gases may better explain the increased MES counts observed in this study. According to Henry's law, the amount of gas that will dissolve in a liquid (eg, blood) at a given temperature (eg, body temperature) is almost directly proportional to the partial pressure of that gas. ${ }^{35}$ At hyperbaria of 1.75 and 2.5 bar, the partial pressures and arterial concentrations of carbon dioxide and water vapor will remain stable, ${ }^{35}$ suggesting that these gases are not the cause of increased MES. When alveolar partial pressure of oxygen rises to values of about $400 \mathrm{~mm} \mathrm{Hg}$, which occurs at $2.5 \mathrm{bar}$, the maximum oxygen saturation of hemoglobin can never increase above $100 \% .{ }^{35}$ Thus, the saturation of hemoglobin would increase by only $3 \%$ because $97 \%$ of the hemoglobin is already combined with oxygen. ${ }^{35}$ Furthermore, only a small amount of oxygen will dissolve in the blood. ${ }^{35}$ These findings suggest that raised arterial partial pressure and concentration of oxygen is an unlikely cause of increased MES counts found at hyperbaria. At atmospheric pressures of 1.75 and 2.5 bar, the partial pressure and concentration of nitrogen will increase. ${ }^{17,28,35}$ Therefore, it is assumed that hyperbaria increases the amount of dissolved nitrogen and thus cavitation bubbles, leading to an increased number of MES. This process seems to be only partially counteracted by the reduced number of cavitation bubbles formed during hyperbaria.

Obviously, the results of the present study cannot be extrapolated to all MES detected in patients with prosthetic heart valves. It appears probable that a portion of these signals is caused by formed embolic material. Currently, no method is able to distinguish between gaseous and formed 
embolic material. Although simultaneous insonation with 2 carrier frequencies, as reported by Brucher and Russell, ${ }^{36}$ appears promising, this approach remains to be evaluated. Such distinction would be of particular interest, because it appears possible that the counts of formed MES in prosthetic valve carriers could serve as a surrogate marker concerning individual stroke risk or efficacy of oral anticoagulation. Previous studies examining the clinical significance of MES were potentially flawed through the high counts of MES caused by gaseous emboli and thus unable to provide valid results.

The interobserver agreement in this study was higher than in previously reported ones, partially due to the fact that both observers were trained in the same laboratory. Additionally, MES in patients with prosthetic heart valves have a higher intensity than those detected in patients with native embolic sources ${ }^{37}$ and are thus easier to distinguish.

In conclusion, the present study provides further evidence for gaseous bubbles as underlying embolic material of part of the detected MES in patients with prosthetic heart valves. The portion of MES caused by formed embolic material remains to be evaluated.

\section{References}

1. Akins CW. Long-term results with the Medtronic-Hall valvular prosthesis. Ann Thorac Surg. 1996;61:806-13.

2. Kuntze CE, Ebels T, Eijgelaar A, Homan van der Heide JN. Rates of thromboembolism with three different mechanical heart valve prostheses: randomised study. Lancet. 1989;1:514-7.

3. Nakano K, Koyanagi H, Hashimoto A, Kitamura M, Endo M, Nagashima M, et al. Twelve years' experience with the St. Jude Medical valve prosthesis. Ann Thorac Surg. 1994;57:697-702.

4. Rodler SM, Moritz A, Schreiner W, End A, Dubsky P, Wolner E. Fiveyear follow-up after heart valve replacement with the CarboMedics bileaflet prosthesis. Ann Thorac Surg. 1997;63:1018-5.

5. Torregrosa S, Gomez-Plana J, Valera FJ, Caffarena J, Maronas JM, Garcia-Sanchez F, et al. Long-term clinical experience with the Omnicarbon prosthetic valve. Ann Thorac Surg. 1999;68:881-6.

6. Fuster V, Dyken ML, Vokonas PS, Hennekens C. Aspirin as a therapeutic agent in cardiovascular disease. Circulation. 1993;87:659-75.

7. Stein B, Fuster V, Halperin JL, Chesebro JH. Antithrombotic therapy in cardiac disease: an emerging approach based on pathogenesis and risk. Circulation. 1989;87:1501-13.

8. Nicholls SC, Glickerman DJ, Lam AM. Cerebral embolisation: transcranial Doppler monitoring in the swine model [abstract]. Stroke 1992; 23:473.

9. Spencer MP. Detection of cerebral arterial emboli. In: Newell DW, Aaslid R, editors. Transcranial Doppler. New York: Raven; 1992. p. 215-30.

10. Babikian VL, Hyde C, Pochay V, Winter MR Clinical correlates of highintensity transient signals detected on transcranial Doppler in patients with cerebrovascular disease. Stroke. 1994;25:1570-3.

11. Georgiadis D, Lindner A, Manz M, Sonntag M, Zunker P, Zerkowski $\mathrm{HR}$, et al. Intracranial microembolic signals in 500 patients with potential cardiac or carotid embolic source and in normal controls. Stroke. 1997;28:1203-7.

12. Grosset DG, Georgiadis D, Abdulah I, Bone I, Lees KR. Doppler emboli signals vary according to stroke subtype. Stroke. 1994;25:382-4.
13. Siebler M, Nachtmann A, Sitzer M, Rose G, Kleinschmidt A, Rademacher J, et al. Cerebral microembolism and the risk of ischemia in asymptomatic high-grade internal carotid artery stenosis. Stroke. 1995;26:2184-6.

14. Georgiadis D, Denzel A, Lehmann D, Lindner A, Zerkowski HR, Zierz $\mathrm{S}$, et al. Influence of oxygen ventilation on Doppler microemboli signals in patients with artificial heart valves. Stroke. 1997;28:2189-94.

15. Kaps M, Hansen J, Weiher M, Tiffert K, Kayser I, Droste DW. Clinically silent microemboli in patients with artificial prosthetic aortic valves are predominantly gaseous and not solid. Stroke. 1997;28:322-6.

16. Spencer MP. Hyperbaric compression and Doppler-detected microemboli in prosthetic valve patients [abstract]. Cerebrovasc Dis. 1996;6:69.

17. Kizer KW. Scuba diving and dysbarism. St Louis: Mosby; 1995.

18. Baumgartner RW, Mattle HP, Schroth G. Assessment of $\geq 50 \%$ and $<50 \%$ intracranial stenoses by transcranial color-coded duplex sonography. Stroke. 1999;30:87-92.

19. Baumgartner RW. Transcranial color-coded duplex sonography. $J$ Neurol. 1999;246:637-47.

20. Ley-Pozo JA, Ringelstein EB, Willmes K. Noninvasive detection of occlusive disease of the carotid siphon and middle cerebral artery. Ann Neurol. 1990;28:640-7.

21. Von Reutern GM, Von Büdingen HJ. Ultrasound diagnosis of cerebrovascular disease. Stuttgart: Thieme; 1993.

22. Consensus Committee of the Ninth International Cerebral Hemodynamic Symposium. Basic identification criteria of Doppler microembolic signals. Stroke. 1995;26:1123-6.

23. Fleiss JL. Statistical methods for rates and proportions. In: Statistical methods and proportions. 2nd ed. New York: John Wiley; 1981.

24. Berger M, Davis D, Lolley D, Rams J, Spencer MP. Detection of subclinical microemboli in patients with prosthetic heart valves [abstract]. $J$ Cardiovasc Tech. 1990;9:282-3.

25. Georgiadis D, Grosset DG, Kelman AW, Faichnez A, Lees KR. Incidence and characteristics of intracranial microemboli signals in patients with different types of prosthetic cardiac valves. Stroke. 1994; 25:587-92.

26. Georgiadis D, Mallinson A, Grosset DG, Lees KR. Coagulation activity and embolic counts in patients with prosthetic cardiac valves. Stroke. 1994;25:1211-4.

27. Sturzenegger M, Beer JH, Rihs F. Monitoring combined antithrombotic treatments in patients with prosthetic heart valves using transcranial Doppler and coagulation markers. Stroke. 1995;26:63-9.

28. Radermacher P, Falke KJ, Park YS, Ahn DW, Hong SK, Qvist J, et al. Nitrogen tensions in brachial vein blood of Korean scuba divers. J Appl Physiol. 1992;73:2592-5

29. Graf T, Fischer H, Reul H, Rau G. Cavitation potential of mechanical heart valve prostheses. Int J Artif Organs. 1991;14:169-74.

30. Lamson TC, Stinebring DR, Deutsch S, Rosenberg G, Tarbell JM. Realtime in vitro observation of cavitation in a prosthetic heart valve. ASAIO Trans. 1991;37:M351-3.

31. Wu ZJ, Wang Y, Hwang NH. Occluder closing behavior: a key factor in mechanical heart valve cavitation. J Heart Valve Dis. 1994;3(Suppl 1): S25-33.

32. Rayleigh OM. On the pressure developed in a liquid during the collapse of a spherical cavity. Phil Mag. 1917;34:94-8.

33. Tomita Y, Shima A. Mechanism of impulsive pressure generation and damage pit formation by bubble collapse. J Fluid Mech. 1986;13:535-64.

34. Biancucci BA, Deutsch S, Geselowitz DB, Tarbell JM. In vitro studies of gas bubble formation by mechanical heart valves. J Heart Valve Dis. 1999;8:186-96.

35. Guyton AC. Textbook of medical physiology. Philadelphia: WB Saunders; 1986.

36. Brucher R, Russell D. Improved discrimination of microembolic events by combining dual frequency Doppler with wavelet transformation [abstract]. Cerebrovasc Dis. 1999;9(Suppl 2):37.

37. Grosset DG, Georgiadis D, Kelman AW, Lees KR. Quantification of ultrasound emboli signals in patients with cardiac and carotid disease. Stroke. 1993;24:1922-4. 